\title{
COMPLEMENTARY TREE PAIRED DOMINATION VERTEX CRITICAL GRAPHS
}

\author{
A. Meenakshi ${ }^{1}$, J. Baskar Babujee ${ }^{2}$ \\ ${ }^{1}$ Department of Mathematics \\ S.K.R. Engineering College \\ Chennai, 600123, INDIA \\ ${ }^{1}$ Anna University \\ Chennai, 600025, INDIA \\ ${ }^{2}$ Department of Mathematics \\ Anna University, MIT Campus \\ Chennai, 600044, INDIA
}

\begin{abstract}
A dominating set $S \subseteq V$ is a paired dominating set if the induced sub graph $\langle S\rangle$ has a perfect matching. The minimum cardinality of a paired dominating set in $G$ is called the paired domination number of $G$ and is denoted by $\gamma_{p r}(G)$. The graph $G$ is paired domination vertex critical if for every vertex $v$ of $G$ that is not adjacent to a vertex of degree one, $\gamma_{p r}(G-v)<\gamma_{p r}(G)$. If $G$ is $\gamma_{p r}$-vertex critical and $\gamma_{p r}(G)=k$, then we say that $G$ is $k-\gamma_{p r}$-vertex critical. In this paper we introduce the concept complementary tree paired domination vertex critical graph and also we present some upper and lower bounds of it. Furthermore, we construct the complementary tree paired domination vertex critical graph.
\end{abstract}

Key Words: graph, paired domination, complementary tree, vertex critical graph

\section{Introduction}

Let $G(p, q)$ be a simple undirected graph with $p$ vertices and $q$ edges. The set

Received: $\quad$ August 30, 3015

Revised: $\quad$ November 23, 2016

Published: November 30, 2016

$\S_{\text {Correspondence author }}$ (c) 2016 Academic Publications, Ltd. url: www.acadpubl.eu 
of vertices is denoted by $V(G)$; the set of edges by $E(G)$. Order of $G$ is denoted by $|G|$ as a symbol for the cardinality of $V(G)$. The degree, neighborhood and closed neighborhood of a vertex $v$ in the graph $G$ are denoted by $d(v), N(v)$ and $N[v]=N(v) \cup\{v\}$ respectively. For a subset $S$ of $V(G), N(S)$ denotes the set of all vertices adjacent to some vertex of $S$ in $G$ and $N[S]=N(S) \cup S$. Let $\langle S\rangle$ and $G-S$ denote the sub graph of $G$ induced by $S$ and $V(G)-S$ respectively. The minimum degree and maximum degree of the graph $G$ are denoted by $\delta(G)$ and $\Delta(G)$ respectively. A vertex of degree one is called a leaf or end vertex and its neighbor is a support vertex. The set of support vertices in $G$ is denoted by $S(G)$. Two vertices at maximum distance apart in $G$ are called diametrical vertices of $G$.

We generally follow graph terminology and notation from [8]. The concept of complement of a graph was defined by Bondy and Murty [1]. Many of the authors introduced the concept related with complements. In particular, Complementary perfect dominating set was introduced by Paulraj Joseph. J and Mahadevan. G [7]. The concept Complementary nil domination number of a graph was introduced by T. Tamizhchelvam and Robin Chelladurai [6]. A paired dominating set $S \subseteq V(G)$ is said to be a complementary tree paired dominating set if the induced sub graph $V-S$ is a tree. The minimum cardinality of a complementary tree paired dominating set (CTPD) of $G$ is called the complementary tree paired domination number of $G$ and is denoted by $\gamma_{c t p d}(G)$ and the corresponding set is $\gamma_{c t p d}$ set of $G$. This concept was introduced by A. Meenakshi and J. Baskar Babujee [4]

A set $S \subseteq V$ is a dominating set if for every vertex $v$ in $V-S$, there exists a vertex $u$ in $S$ such that $v$ is adjacent to $u$. The minimum cardinality of a dominating set in $G$ is called the domination number of $G$ and is denoted by $\gamma(G)$. A matching $M$ in a graph is a set of independent edges in $G$. The number of edges in a maximum matching of $G$ is called the matching number of $G$ which denote by $\alpha^{\prime}(G)$. A vertex of $G$ incident with an edge of the matching $M$ is said to be matched by $M$ or $M$-matched. A dominating set $S \subseteq V$ is a paired dominating set if the induced sub graph $\langle S\rangle$ has a perfect matching. The minimum cardinality of a paired dominating set in $G$ is called the paired domination number of $G$ and is denoted by $\gamma_{p}(G)$. This concept was introduced by Haynes and Slater [8]. Two vertices joined by an edge of $M$ are said to be paired and are called partners in $S$.

The graph $G$ is a paired domination vertex critical if for every vertex $v$ of $G$ that is not adjacent to a vertex of degree one, $\gamma_{p r}(G)>\gamma_{p r}(G-v)$. If $G$ is $\gamma_{p r}$-vertex critical and $\gamma_{p r}(G)=k$, then we say that $G$ is $k-\gamma_{p r}$-vertex critical. This concept was first studied by Edwards [3]. Likewise in this paper we have 
introduced the concept complementary tree paired domination vertex critical graph and it is denoted by $\gamma_{c t p d}$-vertex critical. If $G$ is $\gamma_{c t p d}$-vertex critical and $\gamma_{c t p d}(G)=k$, then we say that $G$ is $k$ - $\gamma_{c t p d}$-vertex critical. We present the characterization of complementary tree paired domination (CTPD) vertex critical graph and also we construct the CTPD vertex critical graph from two smaller ones.

\section{Complementary Tree Paired Domination Vertex Critical Graphs}

Definition 1. The graph $G$ is said to be a complementary tree paired domination vertex critical if for every vertex $v$ of $G$ that is not a cut vertex, $\gamma_{c t p d}(G-v)<\gamma_{c t p d}(G)$. If $G$ is $\gamma_{c t p d}$-vertex critical and $\gamma_{c t p d}(G)=k$ then we say that $G$ is $k-\gamma_{c t p d}$-vertex critical.

For example

(i) $K_{5}$ is $4-\gamma_{c t p d}$-vertex critical, Since $\gamma_{c t p d}(G)=4$ and $\gamma_{c t p d}(G-v)=2$ for every non cut vertex $v \in V(G)$. Therefore $\gamma_{c t p d}(G)>\gamma_{c t p d}(G-v)$. Hence $K_{5}$ is $4-\gamma_{c t p d}$-vertex critical.

(ii) $C_{7}$ is $6-\gamma_{c t p d}$-vertex critical, Since $\gamma_{c t p d}(G)=6$ and $\gamma_{c t p d}(G-v)=4$ for every non cut vertex $v \in V(G)$. Therefore $\gamma_{c t p d}(G)>\gamma_{c t p d}(G-v)$. Hence $C_{7}$ is $6-\gamma_{c t p d}$-vertex critical.

We made some observations

Observation 2. (i) The removal of a vertex can decrease the CTPD number by at most two.

(ii) If $G$ is complete graph of order $n \equiv 1(\bmod 2)$ and $N\left[u_{i}\right]=N\left[v_{j}\right]$ for every $i$ and $j$ then $G$ is $\gamma_{c t p d}$-vertex critical.

In this section we present some characterization of CTPD vertex critical graph.

Theorem 3. The graph $G$ is $\gamma_{c t p d}$-vertex critical if and only if for every non cut vertex $v \in V(G)$ there exists a set $D \subseteq V,|D| \leq \gamma_{c t p d}(G)-2$ such that $D$ is precisely complementary tree paired dominates $G-v$.

Proof. Suppose $G$ is $\gamma_{c t p d}$-vertex critical. Then we have $\gamma_{c t p d}(G-v)<$ $\gamma_{c t p d}(G)-2$ for every non cut vertex $v \in V(G)$. Therefore for every non cut vertex $v \in V(G)$ there exists a set $D$ such that $|D| \leq \gamma_{c t p d}(G)-2, D$ precisely complementary tree paired dominates $G-v$. 
Conversely suppose that for every non cut vertex $v \in V(G)$ there exists a set $D$ such that $|D| \leq \gamma_{c t p d}(G)-2, D$ precisely complementary tree paired dominates $G-v$. Therefore $\gamma_{c t p d}(G-v) \leq|D|<\gamma_{c t p d}(G)$. This implies that $\gamma_{c t p d}(G-v)<\gamma_{c t p d}(G)$. Hence $G$ is $\gamma_{c t p d}$-vertex critical.

Theorem 4. If $G$ is a $\gamma_{c t p d}$-vertex critical then $\gamma_{c t p d}(G-v)=\gamma_{c t p d}(G)-2$ for every non cut vertex $v \in V(G)$.

Proof. Let $G$ be a $\gamma_{c t p d}$ critical graph. Then by theorem 3, for every non cut vertex $v \in V(G)$ there exists a set $D$ with $|D| \leq \gamma_{c t p d}(G)-2$ such that $D$ precisely complementary tree paired dominates $G-v$. Hence $\gamma_{c t p d}(G-v)+2 \geq$ $\gamma_{c t p d}(G)$. The deletion of a vertex decreases the complementary tree paired domination number by at most 2 , which implies that $\gamma_{c t p d}(G-v) \leq \gamma_{c t p d}(G)-2$. Hence $\gamma_{c t p d}(G-v)=\gamma_{c t p d}(G)-2$ for every non cut vertex $v \in V(G)$.

Theorem 5. If $G$ is a $\gamma_{c t p d}$-vertex critical then for every non cut vertex $v \in V(G)$ there exists a $\gamma_{c t p d}$ set $D \subseteq V(G)$ such that $v \in D$

Proof. Let $G$ be a $\gamma_{c t p d}$-vertex critical graph. By theorem 3, for every non cut vertex $v \in V(G)$ there exists set $D^{\prime} \subseteq V(G)$ with $\left|D^{\prime}\right|=\gamma_{c t p d}(G)-2$ such that $D^{\prime}$ precisely complementary tree paired dominates $G-v$. Then the set $D=D^{\prime} \cup\left\{v, v^{\prime}\right\}$ is a CTPD set of $G$ that contains $v$.

Theorem 6. If a connected graph $G$ has any two vertices $u$ and $v$ such that $N(u) \subseteq N(v)$ then $G$ is not $\gamma_{c t p d}$-vertex critical.

Proof. If $v$ is a cut vertex then $\gamma_{c t p d}(G)$ does not exists. Hence $G$ is not $\gamma_{c t p d}$ vertex critical. If $v$ is not a cut vertex then any $\gamma_{c t p d}$ set of $G-v$ contains a vertex in $N(u)$ and which is a CTPD set of $G$. Hence $G$ is not $\gamma_{c t p d}$ vertex critical.

\section{Construction of Complementary Tree Paired Domination Vertex Critical Graphs}

We present the similar construction from Brigham et al [2] that makes it possible to build $\gamma_{c t p d}$ vertex critical graph. Suppose $F$ and $H$ are nonempty graphs. Let $u$ and $w$ be non cut vertices of $F$ and $H$ respectively. Then $(F . H)(u, w: v)$ the coalescence of $F$ and $H$ via $u$ and $w$. 
Theorem 7. Let $F$ and $H$ be any two connected graphs with $\delta(F) \geq 2$ and $\delta(H) \geq 2, u$ and $w$ be any two non cut vertices of $F$ and $H$ respectively and let $G=(F \circ H)(u, w: v)$. Then $\gamma_{c t p d}(F)+\gamma_{c t p d}(H)-2 \leq \gamma_{c t p d}(G) \leq$ $\gamma_{c t p d}(F)+\gamma_{c t p d}(H)$.

Proof. Let $D_{F}$ and $D_{H}$ be $\gamma_{c t p d}$-sets of $F$ and $H$ respectively. If $u \notin D_{F}$ and $w \notin D_{H}$ then $D=D_{F} \cup D_{H}$ is a CTPD set of $G$ with cardinality $\gamma_{c t p d}(F)+$ $\gamma_{c t p d}(H)$. If $u \notin D_{F}$ and $w \in D_{H}$ or $u \in D_{F}$ and $w \notin D_{H}$ then $D=\left(D_{F}-\{u\}\right) \cup$ $\left(D_{H}-\{w\}\right) \cup\{v\}$ is a CTPD set of $G$ with cardinality $\gamma_{c t p d}(F)+\gamma_{c t p d}(H)$. If $u \in D_{F}$ and $w \in D_{H}$, let $D^{v}=\left(D_{F}-\{u\}\right) \cup\left(D_{H}-\{w\}\right)$. Let $M^{v}$ be a maximum matching of $G\left[D^{v}\right]$. Then there exists exactly two vertices $u^{\prime} \in D^{v} \cap N_{F}(u)$ and $w^{\prime} \in D^{v} \cap N_{H}(w)$ such that $u^{\prime}$ and $w^{\prime}$ are not saturated by $M^{v}$. Define $D=D^{v} \cap\left\{u^{\prime \prime}, w^{\prime \prime}\right\}$. Since $M^{v}$ does not have perfect matching there exists two vertices say $u$ " and $w^{\prime \prime}$ such that $u^{\prime \prime} \notin D^{v}$ and $w^{\prime \prime} \notin D^{v}$ but $u$ " $\in N_{F}\left(u^{\prime}\right)$ and $w^{\prime \prime} \in N_{H}\left(w^{\prime}\right)$. Since $\left\langle V(F)-D_{F}\right\rangle$ is a tree and $\left\langle V(H)-D_{H}\right\rangle$ is a tree, $\langle V-D\rangle$ is a tree. Hence $D$ is a CTPD set of $G$ with $|D| \leq \gamma_{c t p d}(F)+\gamma_{c t p d}(H)$. Hence the upper bound is attained.

Let $D$ be a $\gamma_{c t p d}$-set of $G$ and let $D_{F}=D \cap V(F)$ and $D_{H}=D \cap V(H)$, If $v \notin D$ then $v$ is complementary tree paired by $D_{F}$ or $D_{H}$. If $v$ is complementary paired dominated by both of $D_{F}$ and $D_{H}$, then $D_{F}$ and $D_{H}$ are CTPD set of $F$ and $H$ respectively. Hence $\gamma_{c t p d}(G)=|D| \geq \gamma_{c t p d}(F)+\gamma_{c t p d}(H)$. Suppose $v$ is complementary tree paired dominated uniquely by $D_{F}$, then $D_{F}$ is a CTPD set of $F$ and $D_{H} \cup\left\{w, w^{\prime}\right\}$ is a CTPD set of $H$, where $w^{\prime}$ is the partner of $w$. So $|D|+2 \geq \gamma_{c t p d}(F)+\gamma_{c t p d}(H)$, hence $\gamma_{c t p d}(G)+2 \geq \gamma_{c t p d}(F)+\gamma_{c t p d}(H)$. If $v \in D$, then $D_{F}=D \cap V(F) \cup\{u\}$ is a CTPD set of $F$. If $N_{H}(w) \subseteq D$ in $H$, then $D_{H}=$ $D \cap V(H)$ is a CTPD set of $H$. Hence $|D|=\left|D_{F}\right|+\left|D_{H}\right| \geq \gamma_{c t p d}(F)+\gamma_{c t p d}(H)$. If there is a vertex $w^{\prime} \in N_{H}(w)$ such that $w^{\prime} \notin D$, then $D_{H}=D \cap V(H) \cup\left\{w, w^{\prime}\right\}$ is a CTPD set of $H$. Hence $\gamma_{c t p d}(G)+2 \geq \gamma_{c t p d}(F)+\gamma_{c t p d}(H)$, this is the lower bound of $G$.

Theorem 8. [5] Let $F$ and $H$ be a $j-\gamma_{p r}$ vertex critical and $k-\gamma_{p r}$ vertex critical respectively, with minimum degrees at least two, and let $G$ be a graph formed by identifying a vertex of $F$ with a vertex of $H$. If $\gamma_{p r}(G)=j+k-2$, then $G$ is $\gamma_{p r}$-vertex critical.

Theorem 9. [9] Let $F$ and $H$ be connected $\gamma_{p r}$-vertex critical graphs and let $G=F \circ H$. Then $G$ is a $\gamma_{p r}$-vertex critical with $\gamma_{p r}(G)=\gamma_{p r}(F)+\gamma_{p r}(H)-2$ and $\operatorname{diam}(G)=\operatorname{diam}(F)+\operatorname{diam}(H)$.

Theorem 10. Let $F$ be a $i-\gamma_{c t p d}$-vertex critical and $H$ be a $j-\gamma_{c t p d}$-vertex critical with $\delta \geq 2$ and let $G$ be a graph with $\delta(G) \geq 2$ formed by identifying a 
vertex of $F$ with a vertex of $H$. If $\gamma_{c t p d}(G)=i+j-2$ then $G$ is $\gamma_{c t p d}$-vertex critical.

Proof. Let $u \in V(G)$ be any non cut vertex and label the identified vertex $v$. Without loss of generality, $u \in V(F)$. Since $F$ is $i$ - $\gamma_{c t p d}$-vertex critical, $\gamma_{c t p d}(F-u)=i-2$. If $u \neq v$, then every $\gamma_{c t p d}(F-u)$-set can be extended to a CTPD set of $G-u$ by adding to it $\gamma_{c t p d}(H)$. Hence $\gamma_{c t p d}(G-u) \leq i-2+j=$ $\gamma_{c t p d}(G)-2<\gamma_{c t p d}(G)$. If $u=v$ then $v$ is a cut vertex, so $\gamma_{c t p d}(G)$ does not exist.

Theorem 11. [9] Let $F$ and $H$ be two graphs with no isolated vertices and let $G=(F \circ H)(u, w: v)$. If $u \notin S(F)$ and $w \notin S(H)$, then $G$ is $\gamma_{p r}$-vertex critical if and only if both $F$ and $H$ are $\gamma_{p r}$-vertex critical. Furthermore, if $G$ is $\gamma_{p r}$ critical, then $\gamma_{p r}(G)=\gamma_{p r}(F)+\gamma_{p r}(H)-2$.

Theorem 12. Let $F$ and $H$ be two connected graphs with $\delta \geq 2$ and let $G=(F . H)(u, w: v)$. If $u \in V(F)$ and $w \in V(H)$ are not cut vertices then $G$ is $\gamma_{c t p d}$-vertex critical if and only if both $F$ and $H$ are $\gamma_{c t p d}$-vertex critical.

Proof. Assume that $G$ is a $\gamma_{c t p d}$-vertex critical. To prove that $F$ and $H$ are $\gamma_{c t p d}$-vertex critical. Suppose that $F$ is not $\gamma_{c t p d}$-vertex critical, then there exists a non cut vertex $x \in V(F)$ such that $\gamma_{c t p d}(F-x) \geq \gamma_{c t p d}(F)$. Let $D_{x}$ be a $\gamma_{c t p d}$-set of $G-x$. Then

$\left|D_{x}\right|=\gamma_{c t p d}(G)-2=\gamma_{c t p d}(F)+\gamma_{c t p d}(H)-2$. Let $D_{F}=D_{x} \cap V(F)$ and $D_{H}=D_{x} \cap V(H)$. If $x=u$ then $\gamma_{c t p d f}(G-x)=\gamma_{c t p d}(F-u)+\gamma_{c t p d}(H-w) \geq$ $\gamma_{c t p d}(F)+\gamma_{c t p d}(H)-2$ this is a contradiction. If $x \neq u$ and $v \notin D_{x}$ then $D_{H}$ is a CTPD set of $H-w$. Hence $\left|D_{H}\right| \geq \gamma_{c t p d}(H)-2$. If $v$ is paired dominated by $D_{F}$ then $D_{F}$ is a CTPD set of $F-x$. So, $\left|D_{F}\right| \geq \gamma_{c t p d}(F-x) \geq \gamma_{c t p d}(F)$. Since $\left|D_{F}\right|+\left|D_{H}\right|=\gamma_{c t p d}(F)+\gamma_{c t p d}(H)-2,\left|D_{H}\right| \leq \gamma_{c t p d}(H)-2$ which is a contradiction. Hence $v$ is paired dominated by $D_{H}$ and $D_{H}$ is a CTPD set of $H$. So $\left|D_{H}\right| \geq \gamma_{c t p d}(H)$ and $\left|D_{F}\right|=\left|D_{x}\right|-\left|D_{H}\right| \leq \gamma_{c t p d}(F)-2$. Hence $D_{F} \cup\left\{u, u^{\prime}\right\}$ is a CTPD set of $F-x$ with cardinality at most $\gamma_{c t p d}(F)-2$ which is a contradiction to $\gamma_{c t p d}(F-x) \geq \gamma_{c t p d}(F)$. If $x \neq u$ and $v \in D_{x}$ then $\gamma_{c t p d}(G-x)=\gamma_{c t p d}(F-u)+\gamma_{c t p d}(H-w) \gamma_{c t p d}(F)+\gamma_{c t p d}(H)-2$, which is a contradiction. If $v$ is paired dominated by $D_{F}$, then $D_{F}$ is a CTPD set of $F-x$. This is impossible since $\gamma_{c t p d}(F-x) \geq \gamma_{c t p d}(F)$. Thus $v$ is not paired dominated by $D_{F}$. Let $u^{\prime}$ be a neighbor of $u$ in $F$. Then $D_{F} \cup\left\{u, u^{\prime}\right\}$ is a CTPD set of $F-x$ with cardinality at most $\gamma_{c t p d}(F)-2$ which is a contradiction to $\gamma_{c t p d}(F-x) \geq \gamma_{c t p d}(F)$. Hence $F$ is $\gamma_{c t p d}$-vertex critical. Similar way we can prove that $H$ is also $\gamma_{c t p d}$-vertex critical. 
Suppose that $F$ and $H$ are $\gamma_{c t p d}$-vertex critical. We prove that $G=(F . H)(u$, $w: v)$ is $\gamma_{c t p d}$-vertex critical. Let $x$ be any non cut vertex of $V(G)$. If $x \neq v$, say $x \in V(F)-\{u\}$ and $x$ is also a non cut vertex of $F$. Let $D_{F}$ and $D_{H}$ be $\gamma_{c t p d^{-}}$ sets of $F-x$ and $H-w$ respectively. Then $D_{F} \cup D_{H}$ is a CTPD set of $G-x$. Hence $\gamma_{c t p d}(G-x) \leq\left|D_{F}\right|+\left|D_{H}\right| \leq \gamma_{c t p d}(F)+\gamma_{c t p d}(H)-2=\gamma_{c t p d}(G)-2$. If $x=v$ then $v$ is a cut vertex, $\gamma_{c t p d}(G)$ does not exist.

\section{References}

[1] J.A. Bondy Murthy, Graph Theory with Applications, Elsevier Science Publishing Co., Inc, Newyork, N.Y.10017, 1976.

[2] R.C. Brigham, P.Z. Chinn and R.D. Dutton, Vertex domination-critical graphs, Networks, 18 (1988), 173-179.

[3] Edwards, Critically concepts for paired domination in graphs, Masters Thesis, University of Victoria, 2006.

[4] A. Meenakshi and J. Baskar Babujee, On Complementary tree paired domination number (communicated).

[5] Michael A. Henning, Pietermaritzburg, Christina M. Mynhardt and Victoria, The Diameter of Paired-Domination vertex critical graphs, Czechoslovak Mathematical Journal, 58(133) (2008), 887-897.

[6] Mustapha Chellai and Teresa W. Haynes, Total and paired domination numbers of a tree, AKCE J. Gaphs Combin., 1(2) (2004), 69-75.

[7] J. Paulraj Joseph, G. Mahadevan, On complementary perfect domination number of a graph, Acta Ciencia Indica, XXXIM(211) (2006), 847-853.

[8] Teresa W. Haynes, Stephen T. Hedetnimi and Peter J. Slater, Fundamentals of Domination in Graphs, Marcel Decker, 1998.

[9] Xinmin Hou and Michelle Edwards, Paired Domination Vertex Critical Graphs, Graphs and Combinatories, 24 (2008), 453-459. 
